\title{
GEOMETRIC AND MAGNETIC AXES OF THE LHC DIPOLE
}

\author{
M. Bajko, J. Billan, M. Buzio, G. Deferne, P. Ferracin, J. Garcia-Perez, W. Scandale*, E. Todesco, \\ CERN, Geneva, Switzerland.
}

\begin{abstract}
The 15-m long superconducting dipoles of the Large Hadron Collider (LHC) with two-in-one design are curved by about $5 \mathrm{mrad}$ to follow the beam trajectory. They are supported on three cold feet to minimise the vertical sagitta induced by their 35 tonnes weight. The cold masses contain at both ends local multipolar correctors to compensate for the detrimental effect of persistent current during injection. We discuss how we measure and control the geometrical shape of the cold mass and the alignment of the associated correctors and how we identify the magnetic axis of the field-shape harmonics with respect to the expected beam reference orbit. We present results relative to prototype dipoles obtained both at room temperature and in operational conditions at $1.9 \mathrm{~K}$.
\end{abstract}

\section{INTRODUCTION}

The Large Hadron Collider (LHC) under construction at CERN requires $15-\mathrm{m}$ long superconducting dipoles with two-in-one design, in which two counter-rotating proton beams circulate. The active part of each dipole is bent to closely follow the path of the circulating particles and hence provide the necessary mechanical apertures with the minimum coil size. There are two possible choices for the dipole axis: the cold bore axis and the coil axis, determined by mechanical and magnetic measurements respectively. For this reason, we call the first the mechanical axis and the second the magnetic axis. The main scope of this paper is to discuss the relation between the two axes of the LHC dipoles and the beam reference orbit and to clarify to which axis the field-shape harmonics should be referred during magnetic measurements.

In each aperture, the maximum displacement allowed between the beam reference orbit and the cold bore axis is rather small, owing to the beam dynamics requirements during operation. The tolerance range is shaped as a torus of $1-\mathrm{mm}$ radius in the bent part of the cold mass, and as a cylinder of $0.3 \mathrm{~mm}$ radius in the straight ends [1]. Fieldshape distortions are induced by persistent currents during the injection plateau and by yoke saturation and coil deformations due to em forces during collisions. Small correctors welded at the dipole ends are used to compensate their detrimental effect on beam stability. To prevent feed-down effects stemming from misalignments, the dipoles and the associated correctors must be centred with high precision on the beam reference orbit. Systematic alignment errors should not exceed $0.3 \mathrm{~mm}$, whilst random errors can reach $0.5 \mathrm{~mm}(1 \sigma)$ as discussed in [1]. With these constraints, the dipole assembly procedure becomes critical and must be assisted by survey measurements, based on laser trackers and optical moles [1]. The key point is to identify the centre of the two cold bores in the bent cold mass and to reconstruct by a best-fit procedure the optimum path for the two beam orbits along each dipole. The subsequent operations required to complete the dipole assembly, i.e. the welding of the endcorrector supports, of the end-covers, of the support pads, of the interconnection bellows and of the bus-bar holders will use the virtual beam path as the reference for geometric shape. On the other hand, if the field-shape harmonics are referred to the beam reference orbit, the optimum corrector strength required during operation is directly given by the magnetic measurements.

\section{PARAMETERS OF THE LHC DIPOLE}

The LHC ring contains 1232 dipoles, whose main geometrical parameters are listed in Table 1.

Table 1: Geometrical parameters of the LHC dipole

\begin{tabular}{lcc}
\hline & $1.9 \mathrm{~K}$ & $300 \mathrm{~K}$ \\
\hline Magnetic length [m] & 14.300 & 14.343 \\
Bending angle [mrad] & 5.10 & 5.10 \\
Sagitta [mm] & 9.12 & 9.14 \\
Bending radius [m] & 2803.928 & 2812.360 \\
Axes separation [mm] & 194.00 & 194.52 \\
\hline
\end{tabular}

During the LHC operation at $1.9 \mathrm{~K}$, two counterrotating beams follow flat circular trajectories along the $14.3 \mathrm{~m}$ long active part of each dipole. The two orbits are separated by $194 \mathrm{~mm}$, i.e. the nominal distance between the coil axes. In assembly conditions at $300 \mathrm{~K}$, the thermal effect is assumed to leave unchanged the dipole curvature of about $5 \mathrm{mrad}$, and to modify the dipole geometry as indicated in Table 2 . The vertical straightness of the dipole is distorted by about $0.3 \mathrm{~mm}$, between the three supporting pads, by the effect of the self-weight and of the flexural inertia. During assembly, the shape of the cold bore axes is measured in an absolute referential with a 3-D laser tracker and an optical mole, with a precision of $5 \cdot 10^{-6}(1 \sigma)$ relative to the path length of the beam laser. The data are used to fit the reference orbit of the two beams and to establish the horizontal and vertical planes of the cold mass [1].

\section{AXES OF THE COLD-MASS}

A special mole, based on the excitation of a Quadrupole Configured Dipole (QCD), is used to detect the shape of the mechanical and of the magnetic axes of the cold mass

*walter.scandale@cern.ch 
[2]. The mole houses four static tangential coils and a corner cube reflector both positioned in the centre of the mole itself. A mechanical device allows centring the mole in the cold-bore tube. The mechanical axis is measured with a 3-D laser tracker. The magnetic centre of the otherwise homogeneous dipolar field can be observed by powering the two poles of each coil in opposition instead than in series, thereby generating a large skew quadrupole. Its centre can be identified very precisely with the tangential coils by using a low intensity AC current and a synchronous detection.

Four dipoles have been inspected with this method at room temperature. The results are presented using the following conventions. The shape of each axis is referred to the curvilinear path of the reference beam orbit. Horizontal offsets are positive towards the internal side, i.e. the centre of curvature of the dipole. Vertical offsets are positive towards the top direction. In Fig. 1 we show the horizontal shape of the internal tube of the pre-series dipole PSO2.

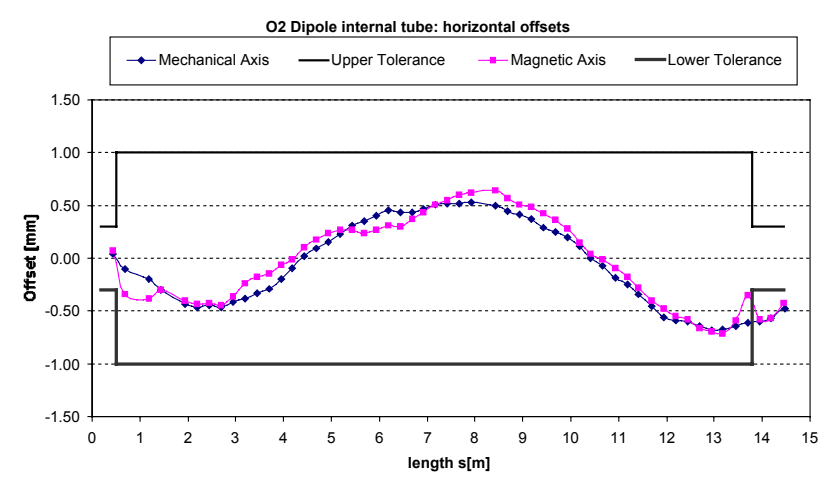

Fig.1 Horizontal axes (internal tube) of the dipole PSO2.

In Table 2 we show the shift between the mechanical and the magnetic axes measured in four dipoles. Average values and the rms fluctuations are computed along the length of each dipole. We also give gives global estimators for the ensemble of the four dipoles, the global rms being computed as the quadratic sum of the individual rms divided by the square root of the dipole number. The global shift is small and well below the radial tolerance of $0.3 \mathrm{~mm}$ required along the dipole-ends. Hence the mechanical and the magnetic axes can be considered equivalent for alignment at room temperature. However, in the cases of P2O1 and PSO1, the average horizontal shift is consistently larger than $3 \sigma$ and its value is at the edge of the $0.3 \mathrm{~mm}$ tolerance range. More dipoles should be measured to decide if this is a trend or a statistical fluctuation.

\section{AXES OF THE CRYO-DIPOLE}

After cryostating, the dipole axes are measured at $1.9 \mathrm{~K}$ with a dedicated travelling probe equipped with a search coil and an optical target, which enables the 3D position
Table 2: Shift of the mechanical and the magnet axes

\begin{tabular}{|l|r|c|c|c|c|c|c|c|}
\hline \multicolumn{9}{|c}{ Mechanical-Magnetic Axis [mm] } \\
\hline & \multicolumn{3}{|c|}{ Internal Tube } & \multicolumn{5}{c}{ External Tube } \\
\cline { 2 - 10 } & Horizontal & \multicolumn{2}{c|}{ Vertical } & \multicolumn{2}{c}{ Horizontal } & \multicolumn{2}{c}{ Vertical } \\
\cline { 2 - 10 } Magne & Ave & $\sigma$ & Ave & $\sigma$ & Ave & $\sigma$ & Ave & $\sigma$ \\
\hline P2O2 & -0.04 & 0.03 & -0.06 & 0.02 & 0.02 & 0.06 & -0.06 & 0.06 \\
\hline P2A2 & 0.09 & 0.03 & -0.02 & 0.02 & -0.05 & 0.03 & -0.08 & 0.03 \\
\hline P2O1 & 0.25 & 0.06 & 0.01 & 0.02 & 0.20 & 0.07 & 0.01 & 0.03 \\
\hline PSO1 & 0.16 & 0.05 & -0.07 & 0.03 & 0.20 & 0.04 & -0.05 & 0.02 \\
\hline Global & 0.12 & 0.05 & -0.04 & 0.02 & 0.09 & 0.06 & -0.04 & 0.04 \\
\hline
\end{tabular}

of the probe axis to be measured by a telescope and a CCD camera. Magnetic measurements can be transferred from the telescope reference frame to the absolute magnet frame by means of a laser tracker survey, which links the two frames via the relative positions of the magnet fiducials and of two reference benches. The estimated precision of the axis co-ordinates in the magnet frame is about $0.17 \mathrm{~mm}(1 \sigma)$.

The probe provides information, which can also be used to refer the cold multipole measurements to the QCD axis. In this way, multipoles can be easily calculated with respect to an arbitrary axis via feed-down. Only about $10 \%$ of the dipoles will be measured with the QCD method in cold condition. All dipoles will be measured at room temperature. The sample population measured with both systems will be used to establish a statistical warmcold correlation, which will enable us to extrapolate the absolute position of the cold QCD axis from warm measurements. The case of the prototype $\mathrm{P} 2 \mathrm{O} 1$ is given as an example in Table 3. The observed shift of the magnetic axis induced by temperature is compatible with the radial tolerance range of $0.3 \mathrm{~mm}$. However, more measurements are required to fully assess if the thermal effect is not detrimental for dipole alignment.

Table 3: Shift of the magnetic axis from room to cryogenic temperature in the dipole $\mathrm{P} 2 \mathrm{O} 1$

\begin{tabular}{ccccc}
\hline & Horizontal shift $[\mathrm{mm}]$ & \multicolumn{2}{c}{ Vertical shift $[\mathrm{mm}]$} \\
\hline & average & $\sigma$ & average & $\sigma$ \\
Ext. bore & -0.10 & 0.26 & -0.22 & 0.10 \\
Int. bore & -0.08 & 0.16 & -0.13 & 0.15 \\
\hline
\end{tabular}

The correlation between QCD and mechanical axis can be investigated also in the cryo-dipole by combining magnetic measurements with fiducialization survey results. In Fig. 2 we show, in an absolute referential, the shape of the horizontal axes of the prototype N2 after cryostating. The measurement is made at room temperature. The average and the rms shift between the mechanical and the magnetic axes is $0.09 \pm 0.11 \mathrm{~mm}$. Similar values are obtained in the other plane and in the other bore. 


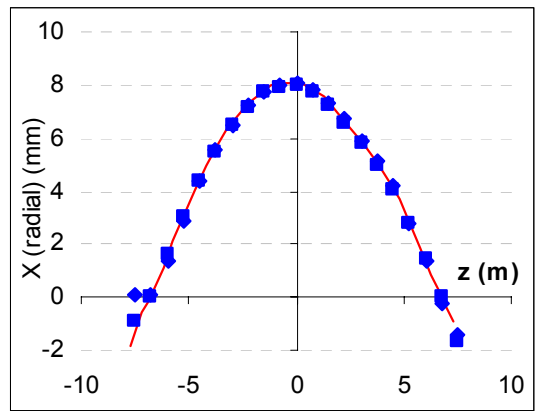

Fig. 3 Case of the dipole P2N2: shape of the geometric (solid line) and the magnetic (dots) axes.

\section{FIELD-SHAPE HARMONICS}

The axis to which field-shape harmonics are referred is evaluated through feed-down of high order harmonics. The method is based on the hypothesis that high order normal and skew even components are zero and that what is observed at order $2 \mathrm{n}$ is due to the feed down of the allowed harmonic $b_{2 n+1}$ according to equation

$$
b_{2 n}=2 n b_{2 n+1} \frac{x_{0}}{R_{r e f}}, \quad a_{2 n}=2 n b_{2 n+1} \frac{y_{0}}{R_{r e f}},
$$

where $x_{0}$ and $y_{0}$ are the horizontal and the vertical displacements between the axis of the rotating coil and the reference axis. For LHC $R_{\text {ref }}$ has been set to $17 \mathrm{~mm}$. The expected values of the high order allowed multipoles, expressed in units of $10^{-4}$, are the followings:

$$
b_{11}=0.60 \quad b_{13}=0.08 \quad b_{15}=0.03 \quad b_{17}=-0.05
$$

In Fig. 2 we show the values of $x_{0}$ evaluated through the feed-down of these four different multipoles, i.e. $b_{11}, b_{13}$, $b_{15}$ and $b_{17}$, in the prototype P2A2. The four estimates coincide within $0.1 \mathrm{~mm}$. In the evaluation of $y_{0}$ one observes some offset between the values given by $b_{11}$ and $b_{17}$, that coincide within $0.1 \mathrm{~mm}$, and the values of $b_{13}$ and $b_{15}$, that may differ by several tenths of $\mathrm{mm}$. Estimates by $b_{11}$ and $b_{17}$ are likely to be more precise. Indeed, the strong systematic value of $b_{11}$ creates a large feed down. On the other hand, $b_{17}$ is less affected by geometric errors of the coil shape, due to the natural power law decay of multipoles given by the Biot-Savart law [3]. For these reasons, $b_{11}$ and $b_{17}$ are used to work out the position of the reference axis. The unexpected behaviour of $b_{13}$ and $b_{15}$ in the $y_{0}$ estimate could be due to a residual systematic component of $a_{12}$ and $a_{14}$ of the order of 0.01 units, that is still not explained. In the hypothesis that the feed-down axis coincides with the coil axis, it would be easy to find the way to refer the field-shape harmonics to the reference beam orbit. This hypothesis will be submitted soon to experimental tests for validation.

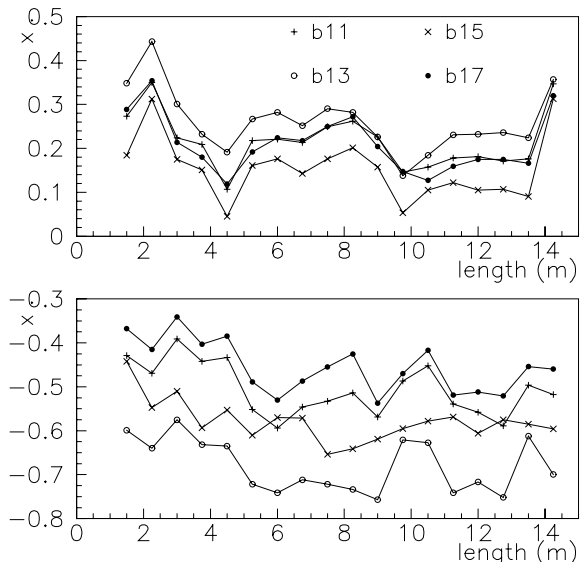

Fig. 3 Axes of high-order field-shape harmonics

\section{CONCLUSION}

We described the methods used in the LHC dipoles to measure the magnetic and the geometric axes, defined as the centre of the coils and of the cold bore respectively. Instruments to measure them both at $300 \mathrm{~K}$ and $1.9 \mathrm{~K}$ are available. The preliminary results refer to prototypes and the first pre-series dipoles. In all cases the shapes of the two axes coincide within a few tenths of $\mathrm{mm}$. Therefore, they can both be used for alignment purpose and this should simplify the assembling procedures and the cryostating operations. This should also allow identifying the reference orbit of the two beams in each dipole by a best fit with the measured shape either of the mechanical or of the magnetic axis. We also pointed out that fieldshape harmonics can be easily referred an axis minimising the feed-down effect of high-order components allowed by the dipolar coil symmetry. The relation of this axis with the beam reference orbit and the magnetic axis is not yet investigated. The issue of the axis stability from dipole manufacture to installation need farther experimental evidences.

\section{REFERENCES}

[1] W. Scandale, M. Bajko, F. Savary 'Geometry and Alignment Requirements for the LHC Main Dipole', 7th EPAC, Vienna, 2000.

[2] J. Billan, et al. 'An AC field static system for measuring the magnetic axis of the LHC superconducting magnets in warm conditions', LHCMMS Int. Note 2000-01, Geneva, 2000.

[3] P. Ferracin, W. Scandale, E. Todesco, R. Wolf Phys.Rev.ST Accel.Beams 3:122403, 2000. 